\title{
Antireflection coatings on plastics deposited by plasma polymerization process
}

\author{
K M K SRIVATSA*, M BERA, A BASU and T K BHATTACHARYA \\ Division of Electronic Materials, National Physical Laboratory, New Delhi 110 012, India
}

MS received 16 August 2007; revised 25 March 2008

\begin{abstract}
Antireflection coatings (ARCs) are deposited on the surfaces of optical elements like spectacle lenses to increase light transmission and improve their performance. In the ophthalmic industry, plastic lenses are rapidly displacing glass lenses due to several advantageous features. However, the deposition of ARCs on plastic lenses is a challenging task, because the plastic surface needs treatment for adhesion improvement and surface hardening before depositing the ARC. This surface treatment is usually done in a multi-stage processexposure to energetic radiations, followed by deposition of a carbonyl hard coating by spin or dip coating processes, UV curing, etc. However, this treatment can also be done by plasma processes. Moreover, the plasma polymerization process allows deposition of optical films at room temperature, essential for plastics. The energetic ions in plasma processes provide similar effects as in ion assisted physical deposition processes to produce hard coatings, without requiring sophisticated ion sources. The plasma polymerization process is more economical than ion-assisted physical vapour deposition processes as regards equipment and source materials and is more cost-effective, enabling the surface treatment and deposition of the ARC in the same deposition system in a single run by varying the system parameters at each step. Since published results of the plasma polymerization processes developed abroad are rather sketchy and the techniques are mostly veiled in commercial secrecy, innovative and indigenous plasma-based techniques have been developed in this work for depositing the complete ARCs on plastic substrates.
\end{abstract}

Keywords. Antireflection coating; polycarbonate; ophthalmic lenses; optical thin film; plasma polymerization.

\section{Introduction}

Antireflection coatings (ARCs) are perhaps the most widely used thin film optical coatings that are applied on the surfaces of optical elements like lenses, prisms, etc used in a variety of optical instruments, including lenses for ophthalmic applications in spectacles. The purpose of an ARC is to minimize the amount of radiant energy that is reflected from the surface of the optical element on which the ARC is applied. Typically, ARCs comprise one or more optical thin films deposited sequentially on the surface of the optical element, to reduce the net reflected light intensity by destructive interference between the light waves reflected/transmitted from the surfaces and interfaces of the films and the substrate (Pulker 1987, 1999; Macleod 2001; Baumeister 2004). ARCs are widely applied on the surfaces of spectacle lenses, because unwanted multiple reflections of incident light from the lens surfaces are thereby largely avoided, and so the visibility of the wearer is improved and a better cosmetic appearance is achieved. The deposition of ARCs on glass lenses has been an ex-

*Author for correspondence (kmk_srivatsa@mail.nplindia.ernet.in) tensively developed subject over several decades, but with plastic lenses increasingly replacing glass lenses in spectacles, the deposition of hard and durable ARCs on plastic lenses has become a very important technological activity over the past decade or so. The most widely used materials for plastic spectacle lenses are CR39 and polycarbonate (PC), as well as polymethyl methacrylate (PMMA) in some cases (Samson 1996; Wikipedia 2006). These plastic materials are not as brittle as glass and thus do not break easily, but they are relatively soft and can be easily scratched. They are also hydrophobic (poor wettability) and film coatings do not readily adhere to their surfaces. Moreover, for the deposition of hard, dense and adherent optical coatings the substrate is usually heated to about $250^{\circ} \mathrm{C}$, to provide the impinging atoms/molecules with sufficient kinetic energy to move to the optimum nucleation sites and adhere well to the surface, but unlike glass plastics cannot be heated above $100^{\circ} \mathrm{C}$. Thus, different techniques are required for the deposition of optical coatings on plastic as compared to glass substrates. In the case of plastic substrates, the required energy is usually provided to the depositing material particles through ionassisted deposition processes - a fairly expensive although well-established technology (Pulker 1987, 1999; Macleod 
2001; Baumeister 2004). Plasma polymerization processes enable the deposition of optical thin films at room temperature, at any rate below $100^{\circ} \mathrm{C}$, and are therefore, especially suitable for plastic substrates (Samson 1996; Shi 1996; Martinu and Poitras 2000). In these processes, the ion energy can be controlled by altering the applied power and bias voltage. The energetic ions in the plasma provide the required energy to the depositing particles, just as in ion-assisted deposition, thereby avoiding the need for the sophisticated ion sources used in ion-assisted physical vapour deposition. The organic liquid precursors whose vapours are decomposed and polymerized by the plasma to deposit the coating are usually much cheaper than the high purity materials that are evaporated or sputtered to deposit the coating. In addition, since most plastic surfaces are hydrophobic, the surface of a plastic substrate has to be made 'wettable' so that the adhesion of the films to the substrate is improved, and a hard coating is required on the surface before the ARC is deposited on it (Samson 1996). Most of the processes in use today for depositing optical coatings like ARCs on plastic substrates are multistep processes: depositing a few nm thick layer of chromium which subsequently oxidizes to form a hard and adherent base layer (Samson 1996), spin- or dip-coating with acrylic resin solutions and curing thermally or by UV exposure (Hozumi et al 1996; Cerac 1997, 1998, 2000; Fraunhofer 2001; Charitidis et al 2004a, b), followed by the deposition of the optical coating by ion-assisted physical vapour deposition or sputtering (Cerac 1998, 2000). Ion implantation techniques (Bhattacharya 1998; Guzman et al 1998) and ion beam sputtering (Sarto et al 1999) have also been used to increase surface hardness and abrasion resistance. However, exposure to plasma (Keil et al 1998; Dahl et al 1999; Seidel et al 1999; Martinu and Poitras 2000; Munzert et al 2003; Lee et al 2004; Muir et al 2006) and plasma polymerization deposition of a carbonyl silica layer (Zajickova et al 1998, 2001; Benitez et al 2000; Kuhr et al 2003; Barrell et al 2004; Kressig et al 2004) offer techniques for achieving surface wettability as well as hardness. These processes, as well as the deposition of the optical coating like ARC itself, can all be carried out by plasma polymerization processes in the same deposition system, by varying the deposition process parameters (the gas and precursor introduced into the chamber, their flow rates, applied power level, bias voltage applied, and so on) at each step. Thus the plasma polymerization deposition process can be more cost-effective, in terms of both equipment as well as materials, than the ion-assisted physical vapour deposition processes. It may be noted, however, that the published literature provides only sketchy details about these processes, all developed abroad and veiled in commercial secrecy.

In the present work, an all-plasma process has been developed indigenously for achieving surface wettability as well as hardness, using a home-made plasma polymerization deposition system. A four-layer ARC comprising alternating thin films of titanium dioxide and silicon dioxide, of different thicknesses, has then been deposited on the surface hardening and adhesion-promoting layer, in the same deposition chamber, to complete the deposition of a hard and durable ARC on the PC substrate. Thus, innovative indigenous plasma-based techniques for depositing composite ARCs on plastic substrates have been developed, which can be useful for indigenous manufacturers of ophthalmic lenses since all coated plastic lenses currently available in India are imported.

\section{Plastic substrates, their characteristics and deposition processes required}

The most commonly used plastic materials in the manufacture of plastic lenses for ophthalmic applications, like spectacle lenses, are CR39 and polycarbonate (PC), although polymethylmethacrylate (PMMA) is also gaining popularity (Samson 1996; Wikipedia 2006).

CR39, specifically poly-diethyleneglycol bisallyl carbonate, is a plastic polymer commonly used in the manufacture of spectacle lenses. CR39 is a trade marked product of PPG Industries, USA, originally developed by Columbia Chemical Co (CR stands for "Columbia Resin'). It is a thermoset polymer, i.e. it is permanently hard when heated and does not soften, and so CR39 lensmaking is an irreversible process. It is harder, stronger and more brittle than most other polymer materials, because of the formation of cross-links between the various constituent functional groups. CR39 is transparent in the visible spectrum and almost completely opaque in the UV range. It has the highest abrasion/scratch resistance of any uncoated optical plastic. CR39 is about half the weight of glass and its refractive index (1.501) is only slightly lower than that of crown glass, making it an advantageous material for spectacle lenses. CR39 is also resistant to most solvents and other chemicals, to gamma radiation, to aging and to material fatigue.

Polycarbonate (PC), specifically polycarbonate of bisphenol-A, is an example of a thermoplastic materialit softens when heated, hardens when cooled, and PC lens-making is a reversible and repeatable process. This property is due to the fact that when it is heated, the effectiveness of the secondary bonds decreases. It is so called because it has functional groups linked together by carbonate groups $(-\mathrm{O}-(\mathrm{C}=\mathrm{O})-\mathrm{O}-)$ in a long molecular chain. It is a very durable material and can be laminated to make bullet-resistant 'glass'. Although it has high impact resistance, it has low scratch resistance and so a hard coating has to be applied on the surfaces of PC spectacle lenses for good durability. The characteristics of polymethyl methacrylate (PMMA) are quite similar but $\mathrm{PC}$ is stronger, though more expensive. PC is highly transparent to visible light and like CR39 it almost completely blocks out UV light. 
The main limitation in using plastics for optical components (Samson 1996) is the softness of their surfaces, which is responsible for low impact and abrasion resistance. These materials are also porous in nature and can absorb water from the atmosphere and expand up to $2 \%$ of their volume, resulting in poor adhesion and even delamination of the coatings on their surfaces. Water exchange in a bulk polymer accelerates surface degradation and can reduce optical performance due to variations in refractive index. The thermal expansion coefficient of plastics is higher than that of inorganic materials like metal oxide films (of which the ARC is composed) by a factor of 10 or so, so that temperature changes may cause weakly adherent coatings to delaminate from the plastic substrate. Plastic lenses develop a much higher electrostatic surface charge than glass lenses, thus attracting dust particles more easily. Besides, plastic lenses are more susceptible to radiation damage.

It is apparent that the deposition of optical coatings like ARCs on plastic substrates is a challenging task. Because of the above mentioned material properties, additional processes are required to make the surface wettable (and therefore, adhesive for deposited coatings), hard and scratch-resistant, as well as to provide it with a waterabsorption barrier, before depositing ARC on it.

As mentioned earlier, most of the commercial processes follow different technologies such as dip-coating and spin-coating techniques (Hozumi et al 1996; Charitidis et al 2004a, b; Cerac 1997, 1998, 2000; Fraunhofer 2001), for increasing surface wettability and surface hardening. However, plasma treatment techniques (Keil et al 1998; Dahl et al 1999; Seidel et al 1999; Munzert et al 2003; Lee et al 2004; Muir et al 2006) and deposition of carbonyl silica layers (Zajickova et al 1998, 2001; Benitez et al 2000; Kuhr et al 2003; Barrell et al 2004; Kressig et al 2004) have been used to achieve surface wettability as well as hardening. Therefore, the plasma process has been adopted and optimized for achieving increased surface wettability and hardness, as well as for the deposition of ARC on the treated plastic surface.

In this paper, $\mathrm{PC}$ has been chosen as the substrate material to deposit multilayer ARCs by the plasma polymerization process. It has been found difficult to procure flat PC and CR39 substrates indigenously. Opticians and lens manufacturers supply only imported CR39 or PC lenses, and those too are not bare lenses but are precoated/treated lenses. Thus, the complete process development for ARCs on plastic lenses cannot be executed on such lenses. In any case, flat substrates are required since curved surfaces as in lenses are not suitable for measurements of the optical transmittance/reflectance of the deposited coatings. Finally, PC sheets have been procured that are about $2 \mathrm{~mm}$ thick (make, GE Lexan), actually meant for architectural purposes but quite transparent though not of ophthalmic quality, and the substrates of $50 \times 50 \mathrm{~mm}$ size have been cut from these sheets.

\section{Experimental: Plasma polymerization deposition system}

A home-made plasma polymerization deposition system, comprising two plane parallel capacitively coupled electrodes in a cylindrical glass deposition chamber, has been designed, fabricated and assembled in-house. RF power can be applied across the electrodes with a RF generator and an auto-matching network, and d.c. bias voltage can also be applied across the electrodes when required. Instead of the commonly used precursors like silane $\left(\mathrm{SiH}_{4}\right)$ gas (for depositing $\mathrm{SiO}_{2}, \mathrm{SiO}_{x} \mathrm{~N}_{y}$ and $\mathrm{Si}_{3} \mathrm{~N}_{4}$ films) and liquid $\mathrm{TiCl}_{4}$ (for depositing $\mathrm{TiO}_{2}$ films), which are toxic, corrosive and difficult to handle, non-toxic and safely handled organic liquid precursors have been used, whose vapours are carried by argon carrier gas into the deposition chamber, along with oxygen gas when required. The details of the deposition system and deposition processes have been reported elsewhere (Srivatsa et al 2008), so they have not been repeated here, but for completeness a schematic diagram of the complete plasma polymerization deposition system has been shown in figure 1.

In order to optimize the deposition parameters for the different films comprising of the composite ARC, individual films have been deposited on silicon $[\mathrm{Si}(100), p$ type] and ophthalmic glass substrates, for the required measurements and characterization of their optical and mechanical properties. The substrates were cleaned following the standard cleaning procedures before loading into the deposition chamber. Prior to each deposition run, the substrates were exposed to argon plasma of $100 \mathrm{~W}$ power at $0.1 \mathrm{mbar}$ pressure for $5 \mathrm{~min}$, to clean the surfaces.

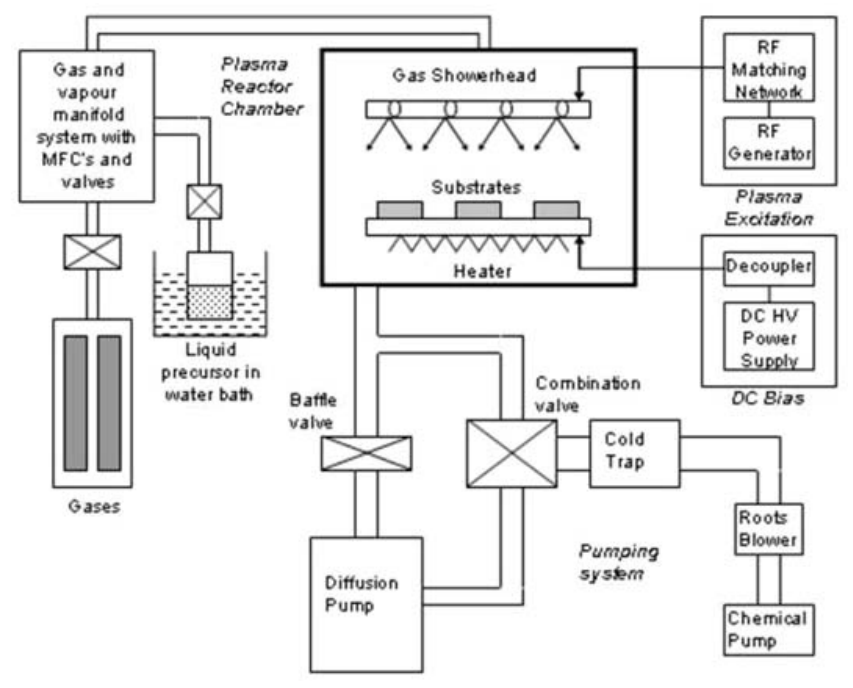

Figure 1. Schematic diagram of plasma polymerization deposition system (Srivatsa et al 2008). 


\section{Results and discussion}

\subsection{Process for making PC surface 'wettable'}

Surface wetting is a phenomenon that makes a film adhere well to the surface. The surface energy should be high for good adhesion. In practice, a surface energy of above 70 dynes/cm is desired for good wettability (Jama et al 1996; Palmers 2000). The wettability of a surface is measured by the contact angle of a water drop on the surface.

Due to its passive nature, the wettability of the plastic surface is very low. To make the surface wettable, it is necessary to increase the surface energy by creating dangling bonds. This can be done by exposing the plastic surface to high energy UV radiation (Martinu and Poitras 2000), but it is difficult to tailor the required energy. Another approach where the required energy can be applied to the surface is the plasma technique, in which the surface is bombarded with energetic ions like $\mathrm{Ar}^{+}$ions (Keil et al 1998; Dahl et al 1999; Seidel et al 1999; Munzert et al 2003; Lee et al 2004). In this technique, energy of $\mathrm{Ar}^{+}$ ions can be tightly controlled by the exciting voltage/ power, or with the external bias. With the applied energy, various bonds on the PC surface will break, and then they are ready to form bonds with adatoms. It is to be noted that the so created dangling bonds have high affinity, and thus last only for a few hours before becoming passive. So, it is necessary to deposit the films immediately after the plasma treatment with $\mathrm{Ar}^{+}$ions for improving wettability. Oxygen plasma treatment of $\mathrm{PC}$, resulting in a low molecular weight material film being formed on the surface of the PC, has also been reported (Muir et al 2006).

The surface treatment adopted in the present work has been the irradiation of the PC surface with $\mathrm{Ar}^{+}$ions in an oxygen environment, rather than the separate exposure to argon and oxygen plasmas reported earlier. This treatment should cause the dangling bonds produced by energetic $\mathrm{Ar}^{+}$ions to form stable bonds with the $\mathrm{O}$ ions. Several runs have been carried out to optimize the practical process parameters to get good surface wettability, which has been checked visually by applying a drop of water on the surface. It has been observed that this exposure to a combination of $\mathrm{Ar}^{+}$and $\mathrm{O}$ ions increases the wettability of the surface and that this wettability lasts longer than in samples exposed to Ar or O plasmas individually. As a matter of fact, there is no need for an additional adhesionpromoting layer on the PC surface since the presence of $\mathrm{O}$ bonds on the surface itself promotes the adhesion of subsequently deposited layers on the surface. Detailed investigations of this process are in progress and will be reported later.

\subsection{Process for hardening of PC surface}

Plastics like PC contain carbonyl groups. So, a plasticlike carbonyl silica film is supposed to adhere well to the plastic substrate due to the presence of carbonyl groups on both sides. Further, carbonyl silica films are known to provide a hard surface (Zajickova et al 1998, 2001; Benitez et al 2000; Kuhr et al 2003; Barrell et al 2004; Kressig et al 2004). A thick carbonyl silica film also acts as sponge to absorb any mechanical shock due to stress effects, etc. (Kuhr et al 2003; Kressig et al 2004). As silica contains oxygen and silicon, subsequently deposited oxide films should adhere better to the carbonyl silica film. Thus a carbonyl silica film acts as a buffer layer between the plastic substrate and ARC comprising of oxide films, with both adhesive as well as hardness properties. Therefore, after making the plastic surface wettable, a carbonyl silica film has been deposited on the plastic surface.

The most widely used organosilicon precursors for the deposition of carbonyl silica films are tetraethoxysilane [TEOS, $\mathrm{Si}\left(\mathrm{OC}_{2} \mathrm{H}_{5}\right)_{4}$ ] and [HMDSO, $\left(\mathrm{CH}_{3}\right)_{3} \mathrm{SiOSi}\left(\mathrm{CH}_{3}\right)_{3}$ ] (Zajickova et al 1998, 2001; Kuhr et al 2003; Barrell et al 2004; Kressig et al 2004). Carbonyl silica films have been deposited using both TEOS and HMDSO. However, since the vapour pressure of HMDSO (42 mbar) is very high as compared to that of TEOS ( 2 mbar), HMDSO liquid can be kept at room temperature and there is no need to heat it to produce adequate vapours, as in the case of TEOS which has to be heated to $60-70^{\circ} \mathrm{C}$ for this purpose. So, HMDSO liquid precursor is easier to handle than TEOS. The results of the deposition process with TEOS and HMDSO have been found to be quite similar.

Initially, carbonyl silicon films were deposited with pure HMDSO, without oxygen, but the films were somewhat absorbing due to excess carbonyl content in the film. Adding oxygen to the HMDSO vapours reduces the carbonyl content in the film, so a little oxygen $(25 \%)$ has been added in the process which resulted in lower optical absorption. Argon has been used as the carrier gas to carry the HMDSO vapours to the deposition chamber.

A typical FTIR spectrum of a $\mathrm{SiO}_{x} \mathrm{C}_{y} \mathrm{H}_{z}$ as-deposited film is shown in figure 2 . In the spectrum one can see the

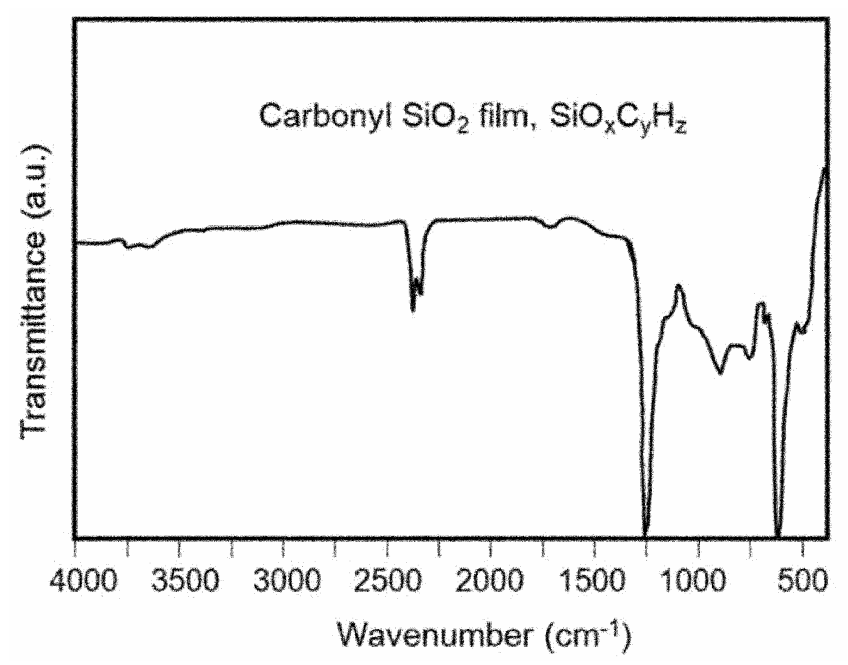

Figure 2. FTIR spectrum of deposited carbonyl silica film. 
presence of different carbon-bearing bands (Barrell et al 2004; Kressig et al 2004). The bands at $1480 \mathrm{~cm}^{-1}$ and $1260 \mathrm{~cm}^{-1}$ are due to $\mathrm{C}-\mathrm{H}$ asymmetrical and symmetrical bending modes in $\mathrm{Si}-\mathrm{CH}_{3}$ groups, respectively. The bands in the $1200-1000 \mathrm{~cm}^{-1}$ interval are due to $\mathrm{Si}-\mathrm{O}-\mathrm{Si}$ and $\mathrm{Si}-\mathrm{O}-\mathrm{C}$ stretching modes. Bands due to $\mathrm{C}-\mathrm{H}$ symmetrical and asymmetrical $\mathrm{C}-\mathrm{H}$ bending in $\mathrm{Si}-\mathrm{CH}_{2}$ groups can be seen at 840 and $769 \mathrm{~cm}^{-1}$, respectively. The FTIR spectrum demonstrates the preservation of some functional groups of the HMDSO molecule. In contrast, $\mathrm{Si}-\mathrm{H}$ and $\mathrm{Si}-\mathrm{O}-\mathrm{C}$ can only be produced by fragmentation of the HMDSO molecule and subsequent recombination of silicon, hydrogen, oxygen and carbon in the discharge (Barrell et al 2004; Kressig et al 2004).

The refractive indices and extinction coefficients, as well as the thicknesses, of the deposited films were measured with a manual null-type research ellipsometer (Rudolph Research, USA). Typically, the refractive index could be determined to within \pm 0.03 and the extinction coefficient estimated to within 0.002 , while the film thickness could be determined to within $\pm 2 \mathrm{~nm}$. The thicknesses of the deposited films were also measured with a TALYSTEP stylus-based instrument (Rank Taylor, UK) to eliminate any uncertainty in the thickness values determined with the ellipsometer.

The typical results obtained for the deposited carbonyl silica films are: deposition rate $\approx 5 \mathrm{~nm} / \mathrm{min}$; refractive index $=1.45-1.55$ (depending on deposition parameters) at $546 \cdot 1 \mathrm{~nm}$; good optical transparency with extinction coefficient $\approx 0.005$; good adhesion and abrasion resistance as per normal adhesive tape/cheesecloth tests.

\subsection{Design of required antireflection coating}

The simplest ARC comprises a single layer of a transparent material whose refractive index is the square root of the refractive index of the substrate (for PC, $\sqrt{1} \cdot 59=1.26$ ) and whose optical thickness (refractive index, $x$, physical thickness) is one-quarter of the wavelength at which the antireflection is required (Pulker 1987, 1999; Macleod 2001; Baumeister 2004). However, this ARC will result in zero reflectance only at this wavelength, while at other wavelengths the reflectance could be more than that of the bare substrate. Moreover, it is very difficult to find a suitable coating material with a refractive index lower than 1.30. Two-layer or three-layer ARCs, comprising of films of two or three materials and with thicknesses of one quarterwave or non-quarterwave at some design wavelength, can provide a low reflectance zone over a short range of wavelengths (Pulker 1987, 1999; Macleod 2001; Baumeister 2004). However, most ARCs applied to spectacle lenses comprise 4-6 layers of two materials with high and low refractive indices (compared to the refractive index of the substrate), in an alternate sequence, with certain non-quarterwave thicknesses, and provide a low reflectance zone covering the visible spectrum (Samson 1996; Cerac 1997, 1998, 2000). Usually, silica $\left(\mathrm{SiO}_{2}\right)$ with a refractive index of about 1.45 and titania $\left(\mathrm{TiO}_{2}\right)$ with a refractive index of about $2 \cdot 2$ are chosen as the low and high refractive index materials, respectively. This is because these materials can be readily deposited in thin film form, are hard and transparent in the visible region, and are compatible with each other in a multilayer stack since they have opposite (tensile and compressive) intrinsic stresses which balance each other to produce low stresses in the composite multilayer (Pulker 1987, 1999; Macleod 2001; Baumeister 2004). Accordingly, a four-layer ARC comprising of alternate layers of $\mathrm{TiO}_{2}$ and $\mathrm{SiO}_{2}$, to be deposited on a carbonyl $\mathrm{SiO}_{2}$ layer on the PC substrate, has been designed using FILMSTAR thin film design software (FTG 1997). It was found that a thickness of $150 \mathrm{~nm}$, and a chosen refractive index of 1.50 , for the carbonyl $\mathrm{SiO}_{2}$ layer yielded best results for the composite ARC deposited on the carbonyl $\mathrm{SiO}_{2}$ layer, and so these values have been chosen for the carbonyl $\mathrm{SiO}_{2}$ layer in the design process. The refractive indices and thicknesses of the various constituent layers that have been obtained by the optimization procedure in the design process are shown in figure 3 . As per the design, the ARC coating is theoretically expected to increase the transmittance from about $90 \%$ for the bare PC substrate to about $96 \%$ for a one-side coated PC substrate, and to about $99 \%$ for a both-sides-coated PC substrate, in the 400-800 nm range. Tolerancing calculations show that only $\pm 2 \%$ variation in film thicknesses in the ARC design can be allowed-if there is more variation, the characteristics of the multilayer ARC stack may degrade appreciably from the optimum characteristics.

\subsection{Deposition of ARC on treated PC surface}

The four-layer $\mathrm{ARC}$ comprising of $\mathrm{SiO}_{2}$ and $\mathrm{TiO}_{2}$ layers of different thicknesses prescribed in figure 3 has been deposited on the surface-hardening layer of carbonyl silica.

\begin{tabular}{|c|c|}
\hline Air & $n=1.0$ \\
\hline $\mathrm{SiO}_{2}$ & $\mathrm{n}=1.45,93 \mathrm{~nm}$ thick \\
\hline $\mathrm{TiO}_{2}$ & $\mathrm{n}=2.20,127 \mathrm{~nm}$ thick \\
\hline $\mathrm{SiO}_{2}$ & $\mathrm{n}=1.45,31 \mathrm{~nm}$ thick \\
\hline $\mathrm{TiO}_{2}$ & $\mathrm{n}=2.20,15 \mathrm{~nm}$ thick \\
\hline Carbonyl $\mathrm{SiO}_{2}$ & $\mathrm{n}=1.50,150 \mathrm{~nm}$ thick \\
\hline
\end{tabular}

Figure 3. Optimized design of composite multilayer coating for an $\mathrm{ARC}$ on a PC substrate. 
As in the case of carbonyl silica films, films of $\mathrm{SiO}_{2}$ have also been deposited by plasma polymerization using TEOS and, more commonly, HMDSO, as the precursor (Mota et al 1995; Durrant et al 1996; Kim 1997; Bulla and Morimoto 1998; Hitchman and Alexandrov 2001). In this work, $\mathrm{SiO}_{2}$ films have been deposited using HMDSO vapours with argon as the carrier gas, with added oxygen to remove the carbonyl content in the deposited film. The FTIR spectrum of a deposited $\mathrm{SiO}_{2}$ film is shown in figure 4. Three characteristic $\mathrm{Si}-\mathrm{O}$ peaks of $\mathrm{SiO}_{2}$ (Bulla and Morimoto 1998; Hitchman and Alexandrov 2001; Kressig et al 2004) at about $1065 \mathrm{~cm}^{-1}, 80 \mathrm{~cm}^{-1}$ and $455 \mathrm{~cm}^{-1}$ are clearly evident. The spectrum also indicates negligible presence of carbonyl content in the film. The typical results for deposited $\mathrm{SiO}_{2}$ films are: deposition rate $\approx 5 \mathrm{~nm} / \mathrm{min}$, refractive index $=1.45-1.47$ at $546.1 \mathrm{~nm}$; good optical transparency with $k \approx 0.002$; good adhesion and abrasion resistance, as per normal adhesive tape/cheesecloth tests.

The most commonly used precursors for $\mathrm{TiO}_{2}$ film deposition by plasma polymerization are TIPT [tetraisopropyl titanate or titanium isopropoxide, $\left.\mathrm{Ti}\left(\mathrm{OC}_{3} \mathrm{H}_{7}\right)_{4}\right]$ and TIBT (tetra isobutyl titanate or titanium tert-butoxide, $\mathrm{Ti}\left(\mathrm{OC}_{4} \mathrm{H}_{9}\right)_{4}$ l (Babelon et al 1998; Battaglin et al 1999; Battiston et al 2000; Hitchman and Alexandrov 2001; Nakamura et al 2001, 2003; Ahn et al 2003). Further, oxygen is usually used to minimize the incorporation of carbonyl groups into the deposited films, which may increase optical absorption in the films. Both TIPT and TIBT have been tried as precursors for the deposition of $\mathrm{TiO}_{2}$ films, with different applied substrate bias voltages and various applied RF power levels. Argon has been used as the carrier gas to carry the reactive vapours into the reaction chamber. Both these precursors have to be heated to about $80^{\circ} \mathrm{C}$ in the flask to generate adequate vapours that are carried into the deposition chamber with the argon carrier gas at about $10 \mathrm{sccm}$. Additionally,

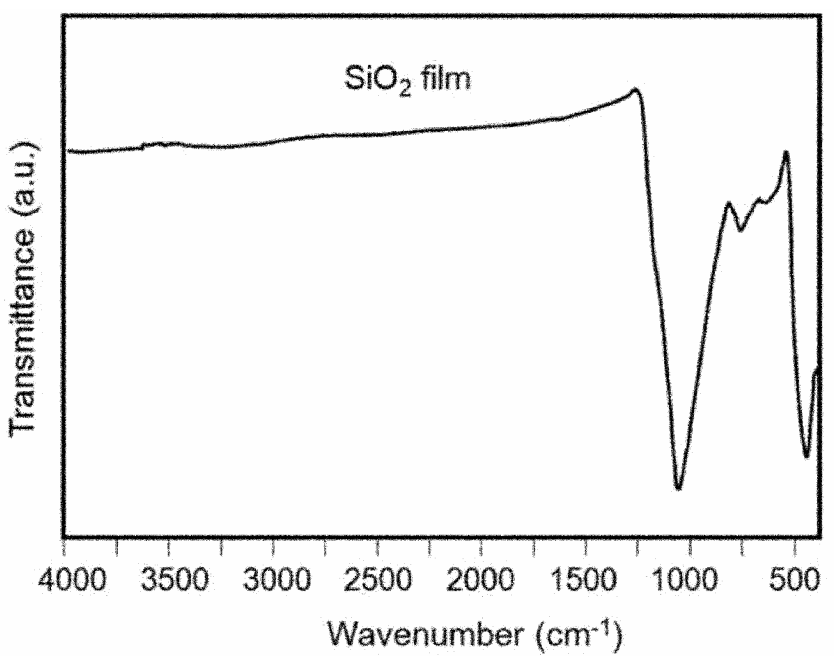

Figure 4. FTIR spectrum of deposited silicon dioxide film (Srivatsa et al 2008). oxygen has also been introduced into the chamber (flow rate, $25-30 \mathrm{sccm}$ ). It has been found that films with better characteristics could be deposited with TIPT rather than TIBT precursor, which tends to form powdery deposits in the deposition chamber and supply lines as well. However, $\mathrm{TiO}_{2}$ films deposited with this oxygen flow rate and using TIPT precursor still contain some residual carbonyl groups and the extinction coefficient is not so low. By increasing the oxygen flow rate to $40 \mathrm{sccm}$, it has been found that hardly any carbonyl groups are present in the deposited films and a low extinction coefficient of about 0.002 or less can be achieved, so an oxygen flow rate of $40 \mathrm{sccm}$ has been used for depositing the $\mathrm{TiO}_{2}$ films for the ARCs. A typical FTIR spectrum of an as-deposited $\mathrm{TiO}_{2}$ film is shown in figure 5. The band extending from $400-800 \mathrm{~cm}^{-1}$ is characteristic of the Ti-O bond (Nakamura et al 2001, 2003). The spectrum also indicates negligible amount of carbonyl content in the film (Nakamura et al 2001, 2003). The typical results for deposited $\mathrm{TiO}_{2}$ films are: deposition rate $\approx 5 \mathrm{~nm} / \mathrm{min}$; refractive index $=$ $2 \cdot 1-2 \cdot 2$ at $546 \cdot 1 \mathrm{~nm}$; good optical transparency with extinction coefficient $\approx 0.002$; good adhesion and abrasion resistance, as per normal adhesive tape/cheesecloth tests.

The deposition rates of the different films have been established after executing several deposition runs for different times and measuring the thicknesses of the deposited films. These deposition rates have been used to decide the deposition time required to deposit a certain film of a particular desired thickness. After depositing the four-layer ARC on one surface of the plasma-treated and carbonyl-silica-coated PC substrate, the ARC was also deposited on the other surface of the substrate, similarly treated beforehand. Figures 6 and 7 show the transmittance versus wavelength characteristics of the deposited composite ARCs, on one side and on both sides of the PC substrate, respectively. Alongside the measured

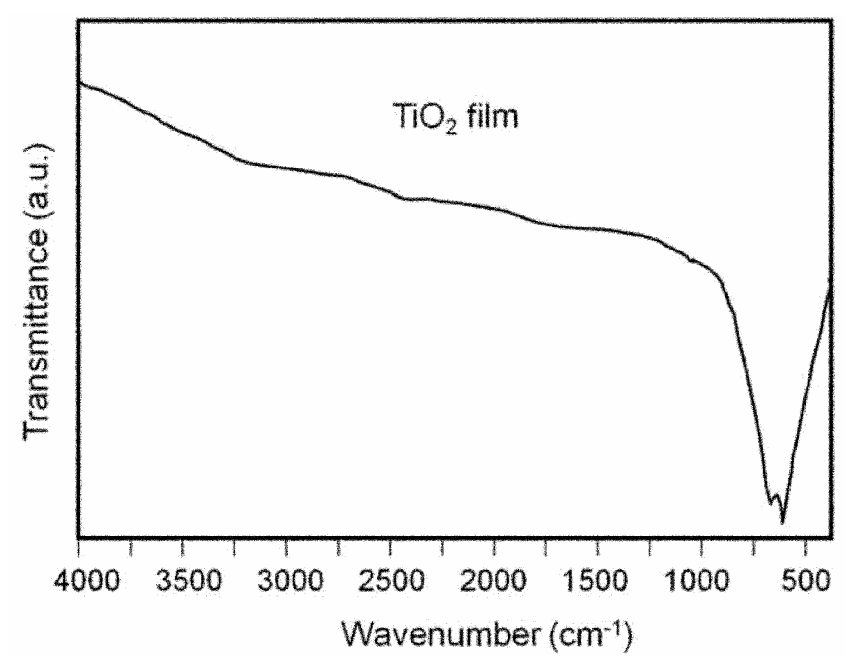

Figure 5. FTIR spectrum of deposited titanium dioxide film (Srivatsa et al 2008) 


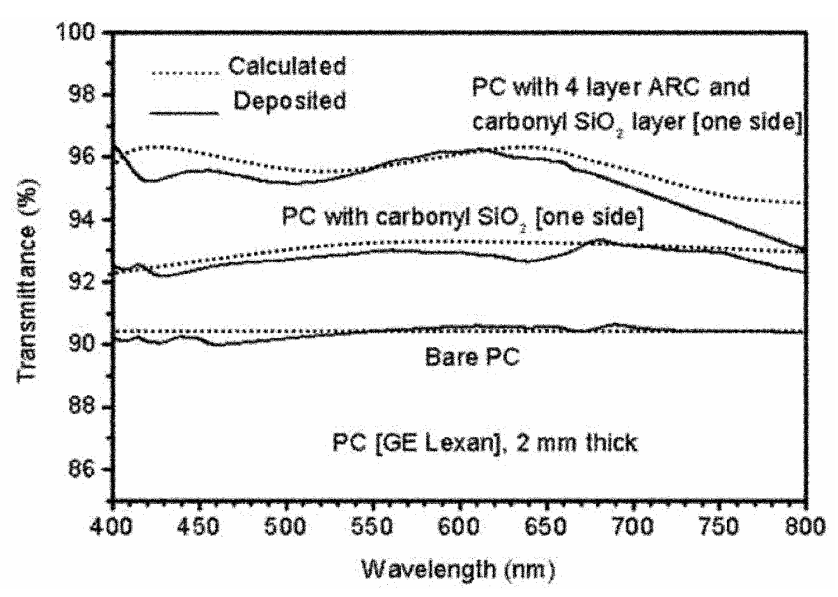

Figure 6. Measured (bold curves) and calculated (dotted curves) transmittance vs wavelength characteristics of a bare PC substrate, a carbonyl silica coated PC substrate, and a fourlayer ARC plus carbonyl silica coated PC substrate (coating of the design shown in figure 3 ), with one side coated.

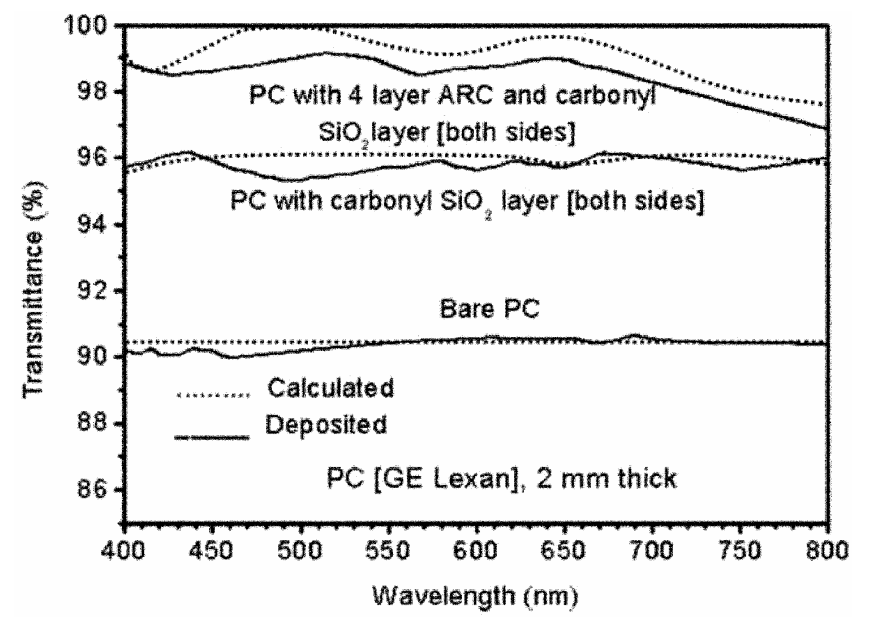

Figure 7. Measured (bold curves) and calculated (dotted curves) transmittance vs wavelength characteristics of a bare PC substrate, a carbonyl silica coated PC substrate, and a fourlayer ARC plus carbonyl silica coated PC substrate (coating of the design shown in figure 3 ), with both sides coated.

characteristic curves for various deposited structures, shown by the bold lines in figures 6 and 7, the calculated characteristics of the various structures are also shown by dotted lines. It can be seen that the measured transmittance characteristics of the deposited composite ARCs match quite well with the calculated characteristics of the designed ARC. The main reason for deviations between the calculated and experimental spectral transmittance characteristics is the uncertainties in the thicknesses of the individual films in the composite ARC. These uncertainties arise because in this work the thicknesses of the individual films are based on the estimated average deposition rates and the correspondingly calculated deposition times of the films, in the absence of any in situ thickness measurement facility in the home-made deposition system. If an in situ spectroscopic ellipsometer is used to take measurements on the growing film during deposition (Martinet et al 1997), the film thicknesses can be measured to within $\pm 0.5 \mathrm{~nm}$ and the film refractive indices can be reproducible to within \pm 0.001 . If an in situ spectroscopic ellipsometer, or atleast a quartz crystal thickness monitor (with RF shielding to ensure stable operation in an RF environment inside the deposition chamber), is installed in the deposition chamber, then the measured characteristics of the composite optical coating can match the calculated characteristics quite closely (Martinet et al 1997).

The deposited composite coating has been cleaned several times by wiping with a cotton cloth moistened with isopropyl alcohol, and also subjected to an adhesive tape peel-off test and a cheesecloth rubbing test. In all these cases, no damage to the surface of the coating was observed visually under a microscope. Further, ARC coated PC samples have been left in the ambient atmosphere for about a month and no degradation or peeling off of the coating has been observed. This indicates that the composite ARC deposited on PC is fairly hard and durable.

\section{Conclusions}

A process has been developed indigenously using a home-made designed and fabricated deposition system, for depositing fairly hard, durable and efficient antireflection coatings on polycarbonate substrates. The process involves making the normally hydrophobic surface of the polycarbonate substrate wettable, followed by the deposition of a surface-hardening layer of carbonyl silica, and finally the deposition of a four-layer antireflection coating comprising alternating layers of silicon dioxide and titanium dioxide, of specified thicknesses. All surface treatment and film deposition processes have been carried out in the same deposition chamber equipped with parallel plate capacitively coupled electrodes (to which RF power at $13.56 \mathrm{MHz}$ is applied), using the plasma polymerization deposition process employing non-toxic and safe organic liquid precursors with argon as the carrier gas, as well as oxygen gas. The spectral transmittance characteristics of the deposited composite antireflection coating, which is fairly hard and durable, compare quite well with the calculated spectral characteristics of the optimum designed coating.

\section{Acknowledgements}

This work was executed under a project [No. DST/ TSG/ME/TTP/2003/57] funded by Technology Systems Group, Department of Science and Technology, Government of India, for which the authors gratefully acknow- 
ledge support. They are thankful to Dr M Kar for providing the spectral transmittance and reflectance traces of the deposited coatings, and to Dr R Mehrotra and Dr M Deepa for providing the FTIR traces for the deposited films. They are grateful to the Director, National Physical Laboratory, New Delhi, for encouragement during this study.

\section{References}

Ahn K -H, Park Y -B and Park D -W 2003 Surf. Coat. Technol. 171198

Babelon P, Dequiedt A S, Mostéfa-Sba H, Bourgeois S, Sibillot P and Sacilotti M 1998 Thin Solid Films 32263

Barrell Y, Creatore M, Schaepkens M, Iacovangelo C D, Miebach T and van de Sanden M C M 2004 Surf. Coat. Technol. 180-181 367

Battaglin C, Caccavale F, Menelle A, Montecchi M, Nichelatti E, Nicoletti F and Polato P 1999 Thin Solid Films 351176

Battiston G A, Gerbasi R, Gregori A, Porchia M, Cattarin S and Rizzi G A 2000 Thin Solid Films 371126

Baumeister P W 2004 Optical coating technology (Washington: SPIE Press) Ch. 2, 4

Benitez F, Martınez E and Esteve J 2000 Thin Solid Films 377378109

Bhattacharya R S 1998 Surf. Coat. Technol. 103-104 151

Bulla D A P and Morimoto N I 1998 Thin Solid Films 33460

Cerac Technical Publications 1997 7(4); 1998 8(3); 2000 10(4); Website: www.cerac.com

Charitidis C, Laskarakis A, Kassavetis S, Gravalidis C and Logothetidis S 2004a Superlattices and Microstructures 36171

Charitidis C, Gioti M, Logothetidis S, Kassavetis S, Laskarakis A and Varsano I 2004b Surf. Coat. Technol. 180-181 357

Dahl S, Rats D, von Stebut J, Martinu L and Klemberg-Sapieha J E 1999 Thin Solid Films 355-356 290

Durrant S F, Mota R P and Bica de Moraes M A 1996 Vacuum 47187

FILMSTAR Thin Film Design Software 1997 (FTG Software, Princeton USA) Website: www.ftg.com

Fraunhofer-Institut fur Angewandte Optik und Finemechanik IOF 2001 Data sheets on ARC and hard coat. Website: www.iof.fraunhofer.de

Guzman L, Celva R, Miotello A, Voltolini E, Ferrari F and Adami M 1998 Surf. Coat. Technol. 103-104 375

Hitchman M L and Alexandrov S E 2001 Interface (Pennington, NJ: The Electrochemical Society) p. 40

Hozumi A, Kato Y and Takai O 1996 Surf. Coat. Technol. 8216
Jama C, Dessaux O, Goudmand P, Mutel B, Gengembre L, Drevillon B, Vallon S and Grimblot J 1996 Surf. Sci. 352354490

Keil M et al 1998 Appl. Surf. Sci. 125273

Kim M T 1997 Thin Solid Films 311157

Kressig U, Gago R, Vinnichenko M, Fernandez-Hidalgo P, Martin-Palma R J and Martinez-Duart J M 2004 Nucl. Instrum. \& Meth. Phys. Res. B219-220 908

Kuhr M, Bauer S, Rothhaar U and Wolff D 2003 Thin Solid Films 442107

Lee J H, Cho J -S, Koh S -K and Kim D 2004 Thin Solid Films 449147

Macleod H A 2001 Thin film optical filters (Bristol and Philadelphia: Inst. of Physics Publishing) 3rd edn, chapters 2, 3

Martinet C, Paillard V, Gagnaire A and Joseph J 1997 J. NonCrystalline Solids 21677

Martinu L and Poitras D 2000 J. Vac. Sci. Technol. A 182619 and references therein

Mota R P, Galvao D, Durrant S F, Bica de Moraes M A, de Oliveira Dantas S and Cantao M 1995 Thin Solid Films 270 109

Muir B W, MacArthur S A, Thissen H, Simon G P, Griesser H J and Castner D J 2006 Surf. Interf. Anal. 381186

Munzert P, Schulz U and Kaiser N 2003 Surf. Coat. Technol. 174-175 1048

Nakamura M, Kato S, Aoki T, Sirghi L and Hatanaka Y 2001 Thin Solid Films 401138

Nakamura M, Makino K, Sirghi L, Aoki T and Hatanaka Y 2003 Surf. Coat. Technol. 169-170 699

Palmers J Jan 2000 Medical device and diagnostic industry magazine (online). Website: www.devicelink.com/mddi

Pulker H K 1987 Coatings on glass (Elsevier: Amsterdam)

Pulker H K 1999 Surf. Coat. Technol. 112250

Samson F 1996 Surf. Coat. Technol. 8179

Sarto F, Alvisi M, Melissano E, Rizzo A, Scaglione S and Vasanelli L 1999 Thin Solid Films 346196

Seidel C, Kopf H, Gostmann B, Vieth T, Fuchs H and Reihs K 1999 Appl. Surf. Sci. 15019

Shi F F 1996 Surf. Coat. Technol. 821

Srivatsa K M K, Bera M, Basu A and Bhattacharya T K 2008 Indian J. Engg. Mater. Sci. (accepted)

Wikipedia The Free Encyclopedia 2006 Website: www. wikipedia.org; Websites like www.allaboutvision.com

Zajickova L, Bursikova V and Janca J 1998 Vacuum 5019

Zajickova L, Bursikova V, Perina V, Mackova A, Subedi D, Janca J and Smirnov S 2001 Surf. Coat. Technol. 142-144 449 